\title{
A Survey of the Use of Empiric Novel Anticoagulants During Investigation of Stroke
}

\author{
Stephen van Gaal, Marc Carrier, Rosendo A. Rodriguez, Mukul Sharma, Ranjeeta Mallick, \\ Grant Stotts, Dar Dowlatshahi
}

Keywords: Atrial fibrillation, anticoagulants, electrocardiography, ambulatory, methodology, survey, stroke

doi:10.1017/cjn.2015.14

Can J Neurol Sci. 2015; 42: 135-137

Atrial fibrillation (AF) is a strong risk factor for ischemic stroke but anticoagulation significantly reduces this risk. Screening for atrial fibrillation is therefore a key component of stroke / transient ischemic attack (TIA) evaluation. Current guidelines recommend a 24-48 hour Holter monitor, but the yield is only 5-7\%. ${ }^{1}$ Extended duration recorders have recently been shown to have a yield of $12.4-16.1 \%$ when used after an initial negative stroke / TIA evaluation (including 24-hour Holter). ${ }^{2,3}$ Given the time needed to obtain these investigations, including the possibility of wait lists with increasing test utilization, it may be necessary to specifically consider how to manage patients pending these investigations.

The empirical use of anticoagulation during this 'peri-investigational' period would offer greater interim protection to the subset of patients with underlying AF. However, due to the increased risk of bleeding of warfarin over acetylsalicylic acid (ASA), empirical anticoagulation with warfarin would likely not be safe. Three novel oral anticoagulants ((NOAC); apixaban, dabigatran, and rivaroxaban) share an approximate 50\% lower risk of intracranial hemorrhage compared to warfarin despite noninferior stroke prevention in $\mathrm{AF}^{4}$ Apixaban specifically demonstrated a risk of hemorrhage comparable to ASA in AVERROES, a trial of ASA vs. apixaban in patients with AF deemed ineligible for anticoagulation with warfarin. ${ }^{5}$ The empirical use of the NOACs during this period might therefore be an efficacious and safe strategy.

As a first step toward exploring the potential value of this, we surveyed academic Canadian neurologists. We asked them to respond to a hypothetical case of stroke with possible underlying $\mathrm{AF}$. Then, in the context of the results of the AVERROES trial, we asked respondents to comment on their appraisal of the empirical use of apixaban in this circumstance.

\section{METHOD \\ Population}

The target population was neurologists affiliated with one of the 16 Canadian academic health centres. A representative from each division of neurology distributed our questionnaire to each of their members by forwarding a generic e-mail invitation. To prevent double counting, we did not use alternative methods of distribution. One reminder was issued, and no incentives were provided.

\section{Survey}

Ethics approval was obtained from the Ottawa Hospital Research Ethics Board. Before the beginning of the study, the investigators reached a consensus on the design and content of the survey questionnaire. Feedback from internal and external vascular neurologists was used to establish the face validity of the survey. The survey consisted of 11 closed-ended questions with multiple-choice responses, three of which used a Likert-type format. In the first three questions, we asked respondents to indicate the diagnostic tests and antithrombotic they would recommend for a patient with a potentially cardioembolic TIA. The next two questions surveyed preferences toward the potential empirical use of apixaban, discussed in the context of the AVERROES clinical trial. The last question required respondents to estimate the equivalent number of hemorrhages that would be needed to cancel out the benefit of an arbitrary number of strokes prevented. Demographic data was collected at the end of the survey. The final survey was programmed in Survey Monkey (www.surveymonkey.com) for electronic distribution. The survey was open for three months (August - October 2013). Survey respondents remained anonymous at all times. The final survey instrument is provided as an online supplement.

\section{Statistics}

Individual-level data was exported directly from the electronic survey application to PASW Statistics (SPSS, Chicago, IL, version 18). Descriptive statistics (frequencies and proportions) were calculated for each demographic and response variable. Post-hoc chi-square statistics were used to assess between-group differences.

\section{RESULTS}

Fourteen out of sixteen academic Neurology Divisions agreed to participate. Sixty-three of 469 (13\%) of neurologists completed

From the Division of Neurology, Department of Medicine, University of Ottawa (SvG, GS, DD); Thrombosis Program, Department of Medicine, University of Ottawa (MC, RAR); Clinical Epidemiology Program, University of Ottawa (MC, DD); Center for Practice Changing Research, Ottawa Hospital (RM); Division of Neurology, Department of Medicine, McMaster University (MS).

Received September 22, 2014. Final Revisions Submitted December 11, 2014. Correspondence to: Stephen van Gaal, Suite C2196, The Ottawa Hospital - Civic Campus, 1053 Carling Avenue, Ottawa Ontario K1Y 4E9. Email: svangaal@ toh.on.ca 


\section{Table 1: Demographic characteristics of survey respondents}

\begin{tabular}{l|c}
\hline Demographic & N (\% of responses) \\
\hline Practice specialization & 57 responses \\
\hline General neurology & $15(26.3 \%)$ \\
\hline Vascular neurology & $22(38.6 \%)$ \\
\hline Other subspecialty & $20(35.1 \%)$ \\
\hline Practice location & 57 responses \\
\hline Academic hospital & $44(77.2 \%)$ \\
\hline Academic office practice & $7(12.3 \%)$ \\
\hline Community hospital & $3(5.3 \%)$ \\
\hline Community office practice & $3(5.3 \%)$ \\
\hline Volume of stroke patients & 57 responses \\
\hline $0-5$ & $16(28.1 \%)$ \\
\hline $6-10$ & $5(8.8 \%)$ \\
\hline $11-20$ & $10(19.3 \%)$ \\
\hline $20+$ & $26(43.9 \%)$ \\
\hline
\end{tabular}

Note: 6/63 missing responses.

the questionnaire, and 57 of the 63 responded to all questions. Demographic information is provided in Table 1.

Responses to the clinical vignette are provided in Table 2. Respondent estimates of the time needed to complete investigations varied with the investigations they requested; $52 \%$ of respondents requesting extended monitoring anticipated waiting at least one month, compared to $19 \%$ of respondents not requesting this investigation $\left(\chi^{2}=8.8, \quad \mathrm{df}=1, \mathrm{p}=0.003\right)$. Recommendation for extended rhythm monitoring did not vary with sub-specialist status $\left(\chi^{2}=0.091, \mathrm{df}=2, \mathrm{p}=0.956\right)$.

Most respondents believed that apixaban would be more effective than ASA for stroke prevention (31/58, 54\%), and as safe or safer with respect to intracranial hemorrhage $(42 / 58,72 \%)$. Respondents were split on their perception of the overall riskbenefit ratio $(17 / 58 ; 29 \%$ in favor of ASA, 20/58; 34\% in favor of apixaban, 21/58; 36\% balanced).

Our respondents identified a number of potential barriers to the empirical use of anticoagulation with apixaban, including lack of evidence $(47 / 58,81 \%)$, colleague perceptions of practice patterns $(38 / 58,65 \%)$ and medicolegal concerns $(28 / 58,48 \%)$. Increased risk of hemorrhage was not an absolute barrier; considering an arbitrary benefit of six ischemic strokes prevented per 100 patients, $34 / 58(59 \%)$ respondents were willing to accept up to two excess intracranial hemorrhages. Half of respondents indicated that they would participate in a clinical trial of empiric anticoagulation versus antiplatelet therapy pending investigation of stroke / TIA with suspected underlying atrial fibrillation $(28 / 57 ; 49 \%)$.

\section{DISCUSSION}

The recent EMBRACE and CYRSTAL-AF trials have demonstrated the efficacy of prolonged rhythm recorders for detecting AF in patients with stroke / TIA and negative initial work-up. ${ }^{2,3}$ It is likely that these recorders will be increasingly used in the investigation of stroke/TIA without an immediately obvious cause. Given the time needed to obtain these investigations, it is now reasonable to explicitly address anti-thrombotic
Table 2: Preferred management strategies in context of a clinical vignette depicting a patient with stroke and suspected underlying atrial fibrillation: recommended investigations (Q1), turn-around time (Q2), and empiric antithrombotic (Q3).

\begin{tabular}{l|c}
\hline Question and responses & N (\% of responses) \\
\hline Requested investigations (Q1, multiple choices) & 63 responses \\
\hline Echocardiogram (TTE/TEE) & $56(88.9 \%)$ \\
\hline Holter monitor (24/48 hour) & $46(73.0 \%)$ \\
\hline Extended rhythm recorder & $27(42.9 \%)$ \\
\hline MRI (+/- MRA) & $18(28.6 \%)$ \\
\hline Expected turn-around time (Q2, single choice) & 63 responses \\
\hline Less than 1 week & $11(17.5 \%)$ \\
\hline $1-2$ weeks & $13(20.6 \%)$ \\
\hline $2-4$ weeks & $17(27.0 \%)$ \\
\hline $1-3$ months & $20(31.8 \%)$ \\
\hline More than 3 months & $2(3.2 \%)$ \\
\hline Empiric antithrombotic (Q3, single choice) & 63 responses \\
\hline ASA & $43(68.3 \%)$ \\
\hline ASA \& clopidogrel & $15(23.8 \%)$ \\
\hline Clopidogrel & $1(1.6 \%)$ \\
\hline Novel oral anticoagulant & $3(4.8 \%)$ \\
\hline Warfarin & $1(1.6 \%)$ \\
\hline
\end{tabular}

Note: No missing responses.

TTE/TEE $=$ transthoracic echocardiography/trans-esophageal echocardiography, MRI = magnetic resonance imaging, $\mathrm{MRA}=$ Magnetic resonance angiography, ASA = acetylsalicylic acid

choice in the peri-investigational period. The empirical use of apixaban during this time may be beneficial, given available data demonstrating a bleeding risk comparable to ASA despite superior protection against stroke and systemic embolism due to $\mathrm{AF}^{5}$

In this study, we sought an appraisal of this strategy. We asked a group of academic Canadian neurologists to respond to a clinical vignette depicting a patient with a TIA and possible underlying $\mathrm{AF}$, and to evaluate a strategy of empiric anticoagulation with apixaban during the peri-investigational period. Although only $29 \%$ of respondents thought that empiric antiplatelet therapy with ASA would be the best option in this circumstance, $95 \%$ reported that this would be what they would recommend to patients. Most respondents cited lack of evidence for the use of apixaban for this indication as an important factor in their decision; as of yet there is no data concerning the use of NOACs for stroke prevention in patients without known AF.

CRYSTAL and EMRBACE-AF have significantly advanced the relevant evidence base by providing reliable estimates of the prevalence of paroxysmal atrial fibrillation in patients with stroke and a negative initial work-up. However, this information is insufficient to answer the question of empiric therapy pending investigation. First, there is no published data establishing the safety or efficacy of any NOAC for the prevention of stroke in patients without atrial fibrillation. Second, it is possible that the risk of stroke due to AF detected in this manner is less than permanent AF or paroxysmal AF detectable through conventional recordings; corresponding benefit may be lower. Additional RCT 
data will be needed before either of these questions can be confidently answered.

To our knowledge, this is the first survey of neurologist attitudes and practices toward the use of anticoagulation as empiric therapy for stroke and suspected underlying AF. As such, it provides a first impression of a group of practicing neurologists on this emerging clinical question. The results of our survey are strengthened by the inclusion of clinicians from across Canada and by the inclusion of a large number of vascular neurologists.

Our survey also has some important limitations. We were only able to achieve a response rate of $13 \%$. This is below average for physician surveys but within reported ranges. We attempted repeat mailings but this had minimal impact. This low response rate may in part be attributable to individual practice patterns, such that some academic neurologists may not manage a significant vascular practice. In the final question of our survey we asked respondents to identify a threshold at which the relative benefit of strokes prevented would be outweighed by hemorrhages caused. We provided simplified estimates for the purpose of relative weighting; these are higher than the likely absolute values and may have introduced bias. We think that this is mitigated by the position of this question as the last of survey and by the clear task of relative weighting against risks. Finally, we appreciate that our survey of academic Canadian neurologists may not be representative of community neurologists or neurologists practicing outside of Canada.

This study suggests that the empiric management of suspected cardioembolic stroke could be an avenue for further research. Our clinicians appeared optimistic that apixaban would be more effective than ASA for the prevention of stroke in patients with suspected underlying AF but would not use it given the lack of evidence. Such work would complement ongoing efforts to determine the prevalence of paroxysmal AF in patients with stroke or TIA.

\section{Conclusion}

Neurologists practicing in academic Canadian centers demonstrated equipoise for the empirical use of apixaban during the investigation of possible cardioembolic stroke / TIA.

\section{Funding}

DD is supported by a Heart and Stroke Foundation of Canada New Investigator Award, and by the University of Ottawa Department of Medicine.

$\mathrm{MC}$ is a recipient of a New Investigator Award from the Heart and Stroke Foundation of Canada and of a Tier 2 Research Chair from the University of Ottawa.

\section{Disclosures}

DD has received speaker honoraria from Boerhinger Ingelheim and Octapharma, and has served on advisory borads for Bayer and BMS/Pfizer.

MS has received speaker honoraria and/or advisory boards for Boerhinger Ingelheim, BMS/Pfizer, Bayer. No ownership interest.

$\mathrm{MC}$ has received speaker honoraria from Boerhinger Ingelheim and Bayer.

\section{SuPPlementary MATERIAL}

To view supplementary material for this article, please visit http://dx.doi.org/10.1017/cjn.2015.14.

\section{REFERENCES}

1. Liao J, Khalid Z, Scallan C, Morillo C, O'Donnell M. Noninvasive cardiac monitoring for detecting paroxysmal atrial fibrillation or flutter after acute ischemic stroke: a systematic review. Stroke. 2007;38:2935-40.

2. Gladstone DJ, Spring M, Dorian P, et al. Atrial fibrillation in patients with cryptogenic stroke. New Engl J of Med. 2014;370: 2467-2477.

3. Sanna T, Diener H-C, Passman RS, et al. Cryptogenic stroke and underlying atrial fibrillation. New Engl J Med. 2014;370: 2478-2486.

4. Chatterjee S, Sardar P, Biondi-Zoccai G, Kumbhani DJ. New oral anticoagulants and the risk of intracranial hemorrhage: traditional and Bayesian meta-analysis and mixed treatment comparison of randomized trials of new oral anticoagulants in atrial fibrillation. JAMA Neurol. 2013;70:1486-90.

5. Connolly SJ, Eikelboom J, Joyner C, et al. Apixaban in patients with atrial fibrillation. New Engl J Med. 2011;364:806-17. 